\title{
Utility function estimation: The entropy approach
}

\author{
Andreia Dionisio $^{\mathrm{a}, *}$, A. Heitor Reis ${ }^{\mathrm{b}, \mathrm{c}}$, Luis Coelho ${ }^{\mathrm{a}}$ \\ ${ }^{a}$ University of Evora, Center of Business Studies, CEFAGE-UE, Largo Colegiais, 2, 7000 Evora, Portugal \\ ${ }^{\mathrm{b}}$ University of Evora, Department of Physics, Portugal \\ ${ }^{\mathrm{c}}$ University of Evora, Evora Geophysics Center, Portugal
}

Available online 4 March 2008

\begin{abstract}
The maximum entropy principle can be used to assign utility values when only partial information is available about the decision maker's preferences. In order to obtain such utility values it is necessary to establish an analogy between probability and utility through the notion of a utility density function. In this paper we explore the maximum entropy principle to estimate the utility function of a risk averse decision maker.
\end{abstract}

(C) 2008 Elsevier B.V. All rights reserved.

Keywords: Maximum entropy; Utility functions; Preferences; Risk aversion

\section{Introduction}

The main goal of this research work is to explore the potential of the maximum entropy principle (ME) to estimate utility functions. In fact, utility functions are one of the most important concepts in decision analysis. They can be estimated empirically using partial information about the agent's preferences and its tolerance about the risk. In this paper we refer to partial information when we only have inferred the utility values based on observed decisions.

The main assumption taken to derive the utility function of an agent, using the ME principle is the correspondence between the concept of equilibrium in physics (statistical) and economics (mechanical). According to some authors (namely Foley [7], Candeal et al. [5], Darooneh [6]) economic equilibrium can be viewed as an asymptotic approximation to physical equilibrium and some difficulties with mechanical picture (economic) of the equilibrium may be eased by considering the statistical (physical) description of it.

In this paper we explore the ME principle to estimate the utility values of a risk averse investor. The rest of the paper is organized as follows. In Section 1 we present a brief discussion of the background theory, namely the ME principle and its applications to economics and more specifically to decision analysis. Section 2 presents the analogy between utility and probability, and utility and entropy. In order to explain in a better way these matters, we show a short example. Finally, Section 3 presents the main conclusions of this study.

\footnotetext{
* Corresponding author.

E-mail addresses: andreia@uevora.pt (A. Dionisio), ahr@uevora.pt (A.H. Reis), lcoelho@uevora.pt (L. Coelho).
} 